Darya Spivakovskaya $\cdot$ Arnold W. Heemink •

Eric Deleersnijder

\title{
Lagrangian modelling of multi-dimensional advection-diffusion with space-varying diffusivities: theory and idealized test cases
}

Received: 1 June 2006 / Accepted: 2 January 2007 / Published online: 24 March 2007

(C) Springer-Verlag 2007

\begin{abstract}
To efficiently simulate the advection-diffusion processes along and across density surfaces, we need to deal with a diffusivity tensor containing off-diagonal elements (Redi, J Phys Oceanogr, 12:1154-1158, 1982). In the present paper, the Lagrangian model, in case of a space-varying diffusivity tensor, is developed. This random walk model is applied for two idealized test cases for which the analytical solutions are known. Results of the testing show that the Lagrangian approach provides accurate and effective solutions of advection-diffusion problems for general diffusivity tensor.
\end{abstract}

Keywords Space-varying diffusivity · Lagrangian model · Pycnocline

\section{Introduction}

Most numerical methods for simulating advection-diffusion processes can be split into three categories as follows:

Responsible editor: Dirk Olbers

Eric Deleersnijder is a research associate with the Belgian National Fund for Scientific Research (FNRS). His contribution to the present study was made in the framework of the development of the Second-generation Louvain-la-Neuve Ice-ocean Model (SLIM, http://www.climate.be/SLIM), which is supported by the Communauté Française de Belgique under the contract ARC 04/09-316.

D. Spivakovskaya $(\bowtie) \cdot$ A. W. Heemink

Department of Mathematical Physics, Delft Institute of Applied

Mathematics (DIAM), Delft University of Technology,

Mekelweg 4,

2628 CD Delft, The Netherlands

e-mail: d.spivakovskaya@ewi.tudelft.nl

Tel.: +31-15-2785179

Fax: +31-15-2787295

E. Deleersnijder

G. Lemaitre Institute of Astronomy and Geophysics (ASTR)

\& Centre for Systems Engineering and Applied Mechanics

(CESAME), Université catholique de Louvain,

Avenue G. Lemaitre 4,

1348 Louvain-la-Neuve, Belgium
Eulerian, Lagrangian, and mixed Eulerian-Lagrangian methods. In Eulerian methods, the transport equation is solved on a fixed spatial grid. The finite element method and finite difference method are primary examples of this class of solution methods. Eulerian methods that have been used for flow simulation were the earliest methods applied to transport modelling, and they are still used commonly today. These methods offer the advantages and convenience of a fixed grid, and they are also easy to implement. However, most Eulerian methods applied to problems with a high initial concentration gradient will lead to serious problems with mass conservation and positiveness (van Stijn et al. 1987; Yang et al. 1998). The examples of the problems that involve sharp concentration front are advection-dominated problems, the problems with deltalike initial concentration, and the problems with general (nondiagonal) space-varying diffusivity tensor. For these problems that exist in many field situations, an Eulerian method is susceptible to excessive numerical dispersion and artificial oscillations (Zheng and Bennett 2002). The effect of numerical dispersion is similar to physical dispersion but is caused by truncation error. When physical dispersion is small or negligible, numerical dispersion becomes a serious problem, leading to the smearing of concentration fronts that should have a sharp appearance (Zheng and Wang 1999). Artificial oscillations are typical for some higher order methods designed to eliminate numerical dispersion and tend to become more severe as the concentration front becomes sharper. It should be noticed that most of the mentioned problems can be reduced by using a sufficiently fine spatial grid and a smaller time step; however, it will increase the computational time significantly.

The Lagrangian approach follows particles through space at every time step. The movement of a particle is modeled with a stochastic differential equation (SDE), which is consistent with the advection-diffusion equation. By simulating the positions of many particles, the advection-diffusion processes can be described. The solution obtained by the random walk method is always mass conservative. Moreover, because the concentration 
of the contaminant is connected with the density probability function of the underlying stochastic process, the solution is always nonnegative. It makes this approach very attractive for a number of applications (Thomson 1987; Kinzelbach 1988; Uffink 1988; Heemink 1990; Dimou and Adams 1993; Zimmermann et al. 2001; Sawford 2001; Proehl et al. 2005; Spivakovskaya et al. 2005).

Lagrangian methods provide an accurate and efficient solution to advection-dominated problems by essentially eliminating the effects of numerical dispersion and artificial oscillations. The random walk method requires relatively little computer storage as compared with finite difference or finite element methods. However, the lack of a fixed grid or fixed coordinate in the Lagrangian method may lead to numerical instability and computational difficulties (Yeh 1990). Another source of numerical error is the interpolation of flow variables in the arbitrary particle location that can lead to local mass balance error and solution anomalies (LaBolle et al. 1996). The random walk solution may also not be smooth if the number of particles is not large enough. As a result, it is difficult to characterize the tailing concentration (Sun 1999). The last problem can be reduced by increasing the number of particles; however, the solution can become too computationally expensive. This disadvantage can be easily compensated by the parallelization of the model. Because of the independence of each realization of the particle movement, the random walk models are extremely suitable for the parallelization. Using parallel processing, the computational time can be reduced significantly.

The Lagrangian approach can be an alternative to the Eulerian methods that involve the steep concentration profile. On the other hand, the Eulerian approach is more suitable for dispersion-dominated problems, for which it provides accurate solutions in reasonable time. The choice of methods depends on the problem under consideration. Sometimes, it is not easy to classify the problem and decide which method should be applied. The mixed Eulerian-Lagrangian methods attempt to combine the advantages of Lagrangian and Eulerian methods. For instance, in the case of a high initial concentration gradient, the Lagrangian approach can be applied to avoid the negativeness of the solution and the loss of mass, and then, the Eulerian method may be used. To eliminate numerical dispersion or artificial oscillations of the solution, the Lagrangian method is used to solve the advective term, and other terms are solved by Eulerian methods. The mixed Eulerian-Lagrangian methods are conceptually attractive and have been widely applied in field applications (Konikow and Bredehoeft 1978; Celia et al. 1990; Yeh 1990; Zhang et al. 1993; Zheng and Wang 1999).

It is common knowledge that large-scale diffusion processes in the ocean occur mostly along isopycnal surfaces, i.e., surfaces of equal density. There is also some diapycnal diffusion. The latter is associated with a diffusion flux orthogonal to isopycnal surfaces. The diapycnal and isopycnal diffusion fluxes are commonly parameterized ă la Fourier Fick-a formulation involving a diffusion tensor that is not diagonal (Redi 1982). As was seen by Beckers et al. (1998, 2000), many Eulerian discretizations of the isopycnal diffusion term yield discrete operators that are not monotonic - a problem that is particularly annoying. The discrete version of the isopycnal mixing parameterization can produce spurious oscillations in the tracer fields, which disagrees with the well-known properties of diffusion operators (Mathieu and Deleersnijder 1998). The Lagrangian method applied to simulate the transport processes along isopycnal surfaces should help avoid spurious oscillations and negative values.

In this paper, random walk schemes associated with nondiagonal diffusivity tensors, whose components vary in space, are established for multidimensional cases (Section 2). The essential theoretical underpinning may be found in Appendix A. These methods are applied for simulating the transport of a passive tracer along isopycnal surfaces (Section 3). The numerical solution is compared with the analytical solution for a linear problem. In Section 4, the random walk algorithm is tested for a onedimensional settling and diffusion model for which key properties of the solution can be derived. Finally, conclusions are drawn in Section 5.

\section{Lagrangian model of multidimensional advection-diffusion}

In an isolated domain of interest, the concentration of a passive tracer can be found from the following boundary value problem in $[0, T] \times \Omega \quad\left(\Omega \subset \mathbb{I R}^{d}\right.$ is domain of interest and $\Gamma$ is its boundary)

$$
\begin{aligned}
& \text { in } \Omega\left\{\begin{array}{l}
\frac{\partial C}{\partial t}=-\nabla \bullet(u C-K \bullet \nabla C) \\
\nabla \bullet \boldsymbol{u}=0
\end{array}\right. \\
& \text { on } \Gamma\left\{\begin{array}{l}
\boldsymbol{u} \bullet \boldsymbol{n}=0 \\
(-\boldsymbol{K} \bullet \nabla C) \bullet \boldsymbol{n}=0,
\end{array}\right. \\
& {[C(t, \boldsymbol{x})]_{t=0}=f(\boldsymbol{x}) .}
\end{aligned}
$$

Here, $\boldsymbol{n}$ denotes the outward unit vector to $\Gamma, \boldsymbol{K}$ is the diffusivity tensor that is symmetric and positive definite (e.g. Deleersnijder et al. (2001)), function $f(\boldsymbol{x})$ represents the initial concentration, and $d$ is the number of space dimensions. In practice, $d=1,2,3$; however, the present Lagrangian approach can be applied for any integer value of $d$. Further, we will assume that $f(\boldsymbol{x})=\delta\left(\boldsymbol{x}-\boldsymbol{x}_{0}\right)$, where $\delta$ denotes the Dirac function. This problem can be also solved with the help of SDEs. The main idea of this approach is to consider the concentration function $C(t, \boldsymbol{x})$ as a transition density function and to interpret Eq. 1 as a Fokker-Planck equation, i.e., the deterministic partial differential equation with regard to transition density 
function. As a result, we can consider the corresponding system of SDEs

$$
\left\{\begin{aligned}
d \boldsymbol{X}(t) & =(\boldsymbol{u}+\nabla \bullet \boldsymbol{K}) d t+\sqrt{2} \boldsymbol{V} d \boldsymbol{W}(t) \\
\boldsymbol{X}(0) & =\boldsymbol{x}_{0}
\end{aligned}\right.
$$

Here, $\boldsymbol{X}(t)$ is the position vector of particles, $d \boldsymbol{X}(t)=$ $\boldsymbol{X}(t+d t)-\boldsymbol{X}(t)$ is a displacement of vector $\boldsymbol{X}$, and $\boldsymbol{K}=\boldsymbol{V} \boldsymbol{V}^{T}, d \boldsymbol{W}(t)$ represents the white noise process. The density function $p(t, \boldsymbol{x})$ of the stochastic process $\boldsymbol{X}$ is the solution of the partial differential equation (Eq. 1). For a detailed explanation of the link between SDEs and partial differential equations, see Appendix A.

In most cases, SDEs like Eq. 2 cannot be solved analytically but only numerically. The numerical solution may be obtained by using one of the numerical procedures, for instance, the Euler scheme (Eq. 48). From here on, we will use the notation $\overline{\boldsymbol{X}}$ for the numerical approximation of the stochastic process $\boldsymbol{X}$.

Random walk models simulate the movement of many particles with the help of the numerical solution of the system of SDEs (Eq. 2) and then, from the obtained sample, construct the probability density function using the methods of nonparametric statistics (see Fig. 1). The resulting function is the solution of the Fokker-Planck equation, which in our case, is the advection-diffusion equation (Eq. 1).

One should be careful when particles are close to the boundaries. Theoretically, the particle cannot cross the boundary; however, because of the discretizations of the stochastic system (Eq. 2), particles can occur outside the domain. To prevent this, we need to take a sufficiently small time step. This procedure is described in Stijnen (2002). The original time step is halved, letting the particle travel two short time steps instead of a single big one. This process is repeated until the

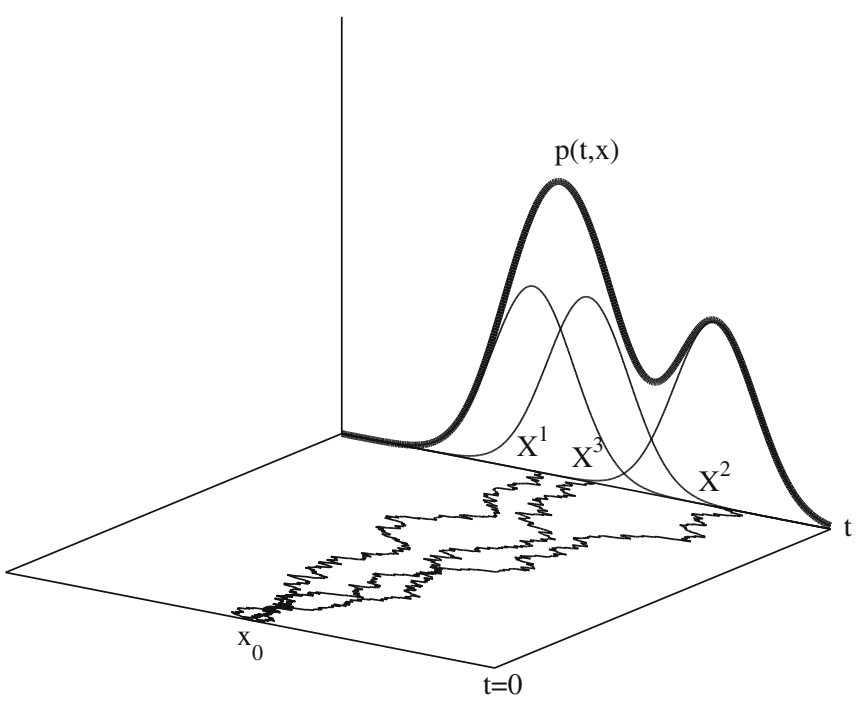

Fig. 1 Kernel estimator particle does not cross the boundary anymore. The result is that the particle trajectory bends along a certain boundary but never crosses it.

It is a common practice to calculate the averages over the grids in the space, i.e., by calculating the number of particles in a box (see, for instance, Riddle 1998). The estimation of the concentration is then obtained by multiplying the number of particles in each box with their mass and dividing the total mass by the volume of the box. This estimator depends on the choice of the boxes: their sizes and the centers of the averaging intervals. This method is ideal if the volume average over such box is exactly what the modeler wants. However, for many cases, this method is not sufficiently accurate and requires much CPU time. On one hand, we cannot choose the number of boxes to be too large; otherwise, the concentration function becomes very irregular or noisy (having large variance). On the other hand, we cannot describe the concentration function in one box more precisely than a constant.

Another method that allows the reduction of the number of particles by an order of magnitude is called kernel estimator (Silverman 1986; Wand and Jones 1995)

$$
\widehat{p}(t, \boldsymbol{x})=\frac{1}{N \lambda^{d}} \sum_{n=1}^{N} \mathcal{K}\left(\frac{\overline{\boldsymbol{X}}^{(n)}(t)-\boldsymbol{x}}{\lambda}\right) .
$$

Here, $N$ is a number of realizations of the stochastic process $\overline{\boldsymbol{X}}(t), \mathcal{K}(\boldsymbol{u})$ is a kernel function (any function that satisfies the condition $\left.\int \mathcal{K}(\boldsymbol{u}) d \boldsymbol{u}=1\right), \lambda$ is a positive number, usually called bandwidth and $\overline{\boldsymbol{X}}^{(n)}(T)$, $n=1, \ldots N$ is a sample from the approximation $\bar{X}$ of the process $\boldsymbol{X}$. One can think of the kernel estimator as spreading of a "probability mass" of size $1 / N$ associated with each data point about its neighborhood. Combining contributions from each data point means that in regions with many observations, the density has a relatively large value and is opposite in regions with only few observations.

Usually the kernel function is chosen to be a probability density function that is symmetric about zero, for instance, the Gaussian

$\mathcal{K}(\boldsymbol{u})=(2 \pi)^{-d / 2} \exp \left(-\frac{1}{2} \boldsymbol{u}^{\mathrm{T}} \boldsymbol{u}\right)$

or Epanechnikov function

$\mathcal{K}(\boldsymbol{u})=\frac{1}{2} v_{d}^{-1}(d+2)\left(1-\boldsymbol{u}^{T} \boldsymbol{u}\right) 1_{\boldsymbol{u}^{T} \boldsymbol{u} \leq 1}$,

where $\nu_{d}=2 \pi^{d / 2} /\{d \Gamma(d / 2)\}$ is the volume of the unit $d$ dimensional sphere, and $\Gamma(x)$ is a Gamma function. This ensures that $\widehat{p}(t, \boldsymbol{x})$ is itself also a density. For example, to construct the kernel estimator shown on Fig. 1, we used the one-dimensional Gaussian kernel. 
So far, we supposed that the probability density function $\widehat{p}(t, \boldsymbol{x})$ satisfies certain smoothing criteria. In particular, it may be easily shown (Wand and Jones 1995) that if the density $\widehat{p}(t, x) \quad x \in \mathrm{IR}$ is twice continuously differentiable, then

$E \widehat{p}(t, x)=p(t, x)+\mathcal{O}\left(\lambda^{2}\right)$.

In practice, however, most densities are discontinuous. For the sake of simplicity, we consider a one-dimensional case. Suppose density $p(t, x)$ is such that $p(t, x)=0$, $x \notin[0,1]$. The continuous operation of the kernel estimator (Eq. 3) will not perform well at the discontinuities. In this case, the kernel estimator overspills the boundaries as it is shown on Fig. 2. Moreover, the estimator (Eq. 3) is not consistent with the true value of the density function at and near the boundaries, and the equation (Eq. 6) is not valid anymore.

As the locations of the boundaries are usually known, the kernel estimator may be adapted to achieve better performance near the boundaries. One of the most natural and simplest way is to reinstate the "missing mass" by reflecting the estimate in the boundary (e.g., Silverman 1986). The kernel function is reflected with regard to the boundary (see Fig. 3). In this way, we can achieve the consistency of the estimator; however, this method still results in a large bias. It is not difficult to show that for the kernel function $\mathcal{K}(u)$ with compact support on $[-1,1]$ (for instance, the Epanechnikov function (Eq. 5) near the boundary $x=0$, the following equation will be valid

$E \widehat{p}(t, x)=p(t, x)+\nu_{\alpha}(\mathcal{K}) p^{\prime}(x) \lambda+\mathcal{O}\left(\lambda^{2}\right)$,

where $x=\alpha \lambda, 0<\alpha<1$ and $\nu_{\alpha}(\mathcal{K})$ are parameters that depend on the kernel function $\mathcal{K}(u)$ and $\alpha$. This formula may be found, for instance, in Jones (1993).

A variety of further modification is possible to achieve a smaller bias. One can think of these boundary modifica-

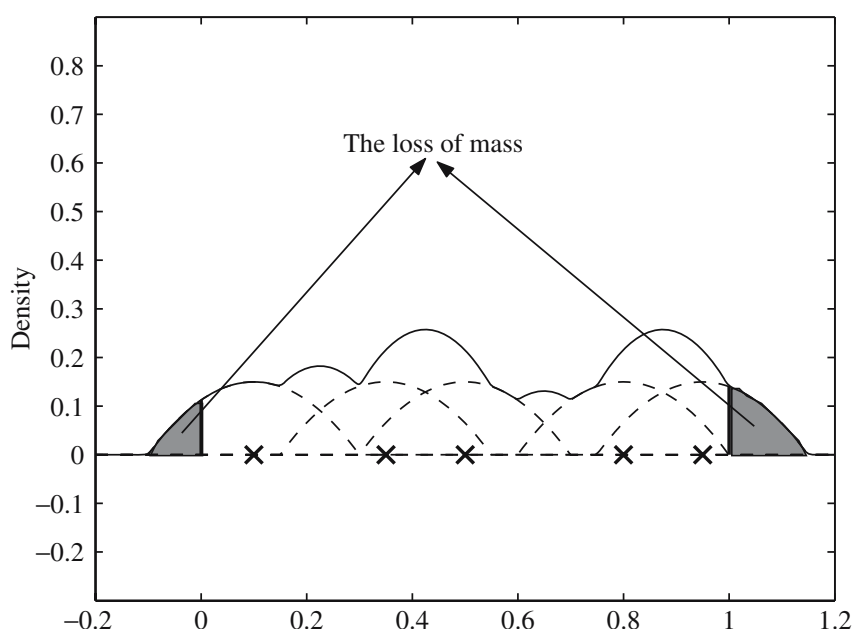

Fig. 2 The loss of mass because of the kernel estimator

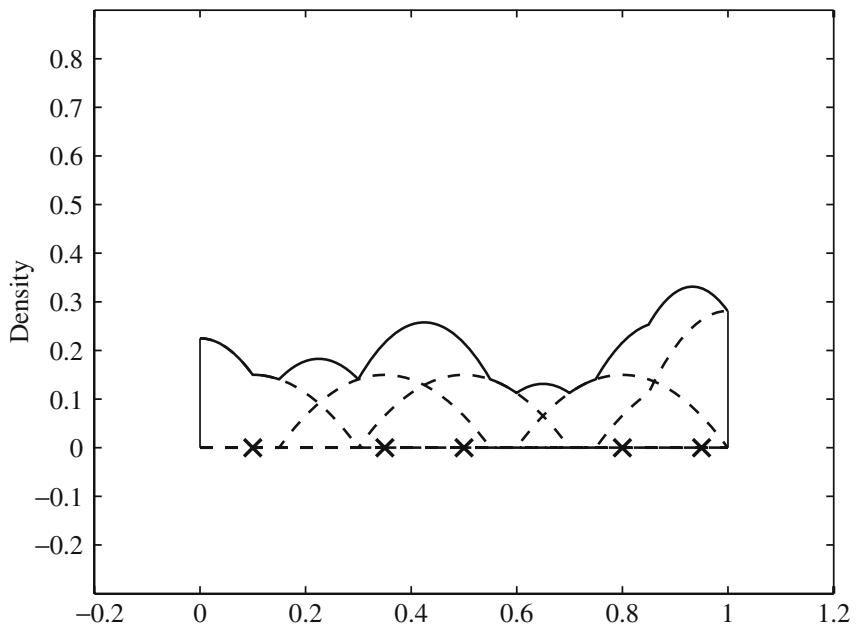

Fig. 3 The transformation of the kernel (Epanechnikov) function

tions in terms of special "boundary kernels" that are different for every $\alpha$. The example of the boundary kernel based on Epanechnikov function is

$$
\begin{aligned}
\mathcal{K}(x) & =6(1+x)(\alpha-x) \frac{1}{(1+\alpha)^{3}} \\
& \left(1+5\left(\frac{1-\alpha}{1+\alpha}\right)^{2}+10 \frac{1-\alpha}{(1+\alpha)^{2}} x\right)
\end{aligned}
$$

This boundary kernel was proposed by Müller (1991). Unfortunately, boundary kernels do not guarantee the positiveness of the numerical solution (see Fig. 4). Besides the described methods, there are many techniques of the kernel transformation in the boundary. An overview of boundary kernels was presented by Jones (1993).

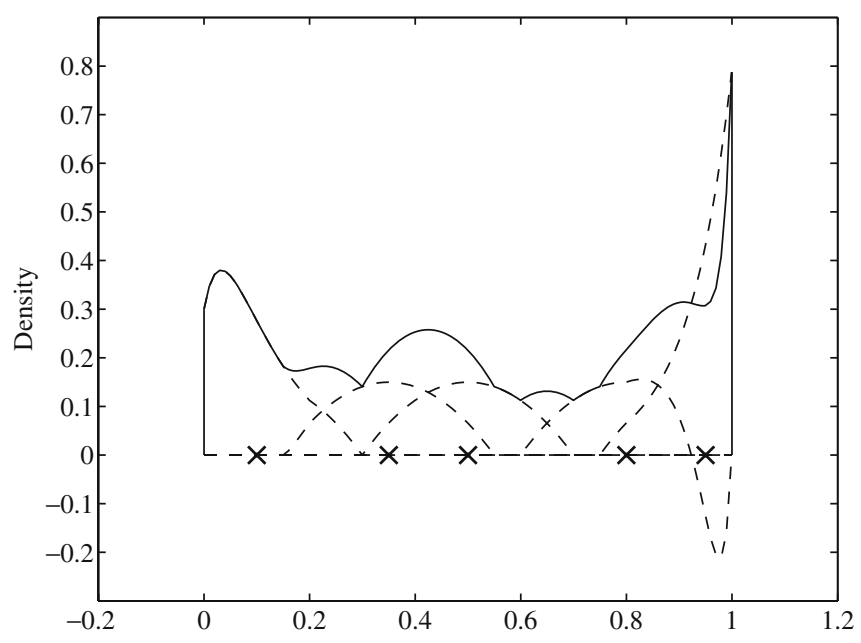

Fig. 4 The kernel estimator transformed by using "boundary kernels" 
The problem of mass loss near the boundary may be easily avoided by using the box estimator. However, the box estimator is less efficient in interior points. Additional information about the advantages and disadvantages of the kernel estimator as compared with the box estimator for particle models can be found in de Haan (1999). Furthermore, for one of the test problems, the two methods are compared, and it can be seen that the kernel estimator gives the better approximation than the box estimator.

The error of the estimator (Eq. 3) can be split into two parts as follows: the error because of the numerical approximation of the system (Eq. 2) and the error because of the kernel estimator. The first error depends on the choice of time step $\Delta t$ (see Appendix A); whereas the second error depends on the number of particles. It is well known (Silverman 1986; Wand and Jones 1995) that the optimal bandwidth is given by

$\lambda_{o p t} \sim N^{-\frac{1}{d+4}}$

and the error because of the kernel estimator is of the following order

$\varepsilon(p) \sim N^{-\frac{2}{d+4}}$

It should be noticed that it is inappropriate to choose a very small time step while the number of particles stays relatively small or opposite to start the numerical simulation for many particles, while the time step is big. It can be proven (Schoenmakers and Heemink 1997) that for the numerical scheme for the SDE of weak order $\beta$ (see Appendix A) and for the kernel estimator, the following choice of parameters $\Delta t$ and $N$

$\Delta t(\varepsilon)=\mathcal{O}\left(\varepsilon^{\frac{1}{\beta}}\right) \quad N(\varepsilon)=\mathcal{O}\left(\varepsilon^{-2-\frac{\mathrm{d}}{2}}\right)$

leads to an error smaller than $\varepsilon$ with a minimum computational time.

\section{Test problem 1: linear two-dimensional iso- and diapycnal diffusion problem}

First, we discuss the Lagrangian method described above for iso- and diapycnal diffusion problems that can be solved analytically. We consider a multidimensional model and suppose that the advective processes can be neglected, and the concentration $C(t, \boldsymbol{x})$ of a passive tracer obeys the following equation:

$\frac{\partial C}{\partial t}=\nabla \bullet(K \bullet \nabla C)$.
If homogeneity can be assumed along one horizontal coordinate, a two-dimensional problem is to be dealt with. For a large-scale ocean model, the formulation of the diffusion model resorts to two diffusivity coefficients, $K^{i}$ and $K^{d}$, which are isopycnal and diapycnal diffusivities, respectively. In the principal axes, the diffusivity tensor reads

$\boldsymbol{K}=\left(\begin{array}{cc}K^{i} & 0 \\ 0 & K^{\mathrm{d}}\end{array}\right)$

The first principal axis is parallel to the isopycnal direction, whereas the other is orthogonal to it. Let $\theta$ denote the angle between the horizontal and the isopycnal direction; in the horizontal-vertical coordinates $(x, z)$, the diffusivity tensor is (Redi 1982; Beckers et al. 1998; Mathieu et al. 1999)

$\boldsymbol{K}=\left(\begin{array}{ll}\cos ^{2} \theta K^{i}+\sin ^{2} \theta K^{\mathrm{d}} & \sin \theta \cos \theta\left(K^{i}-K^{d}\right) \\ \sin \theta \cos \theta\left(K^{i}-K^{\mathrm{d}}\right) & \sin ^{2} \theta K^{i}+\cos ^{2} \theta K^{d}\end{array}\right)$.

The domain of interest is assumed to be infinite,

$-\infty<x<\infty, \quad-\infty<z<\infty$

and the initial concentration is a Dirac impulse, i.e.,

$C(0, \boldsymbol{x})=\delta(\boldsymbol{x}-\mathbf{0})=\delta(x-0) \delta(z-0)$.

It is useful to introduce dimensionless variables. The time and space coordinates are transformed as follows:

$t^{\prime}=\frac{t}{T}, \quad x^{\prime}=\frac{x}{L_{h}}, \quad z^{\prime}=\frac{z}{L_{v}}$,

where $T, L_{h}$, and $L_{\nu}$ denote the appropriate time scale, horizontal length scale, and vertical length scale. It is convenient to define the latter in such a way that

$T=\frac{L_{h}^{2}}{K^{i}}=\frac{L_{v}^{2}}{K^{d}}$.

The ratio of the vertical length scale to the horizontal one, i.e., the aspect ratio, is

$\alpha=\frac{L_{v}}{L_{h}}$.

The concentration is scaled as follows:

$C^{\prime}=\frac{C}{1 /\left(L_{h} L_{v}\right)}$. 
The equation to be solved now reads

$$
\frac{\partial C^{\prime}}{\partial t^{\prime}}=\frac{\partial}{\partial x^{\prime}}\left(K_{x x}^{\prime} \frac{\partial C^{\prime}}{\partial x^{\prime}}+K_{x z}^{\prime} \frac{\partial C^{\prime}}{\partial z^{\prime}}\right)+\frac{\partial}{\partial z^{\prime}}\left(K_{z x}^{\prime} \frac{\partial C^{\prime}}{\partial x^{\prime}}+K_{z z}^{\prime} \frac{\partial C^{\prime}}{\partial z^{\prime}}\right)
$$

with

$$
\begin{aligned}
& K_{x x}^{\prime}=\frac{K_{x x}}{L_{h}^{2} / T}=\cos ^{2} \theta+\alpha^{2} \sin ^{2} \theta, \\
& K_{x z}^{\prime}=\frac{K_{x z}}{L_{h} L_{v} / T}=\left(\alpha^{-1}-\alpha\right) \sin \theta \cos \theta, \\
& K_{z x}^{\prime}=\frac{K_{z x}}{L_{h} L_{v} / T}=\left(\alpha^{-1}-\alpha\right) \sin \theta \cos \theta, \\
& K_{z z}^{\prime}=\frac{K_{z z}}{L_{v}^{2} / T}=\cos ^{2} \theta+\alpha^{-2} \sin ^{2} \theta .
\end{aligned}
$$

The initial condition is

$$
C^{\prime}\left(0, \boldsymbol{x}^{\prime}\right)=\delta\left(\boldsymbol{x}^{\prime}-\mathbf{0}\right)=\delta\left(x^{\prime}-0\right) \delta\left(z^{\prime}-0\right) .
$$

From here on, only dimensionless variable quantities will be dealt with. Therefore, for the sake of simplicity, the primes will be dropped.

The general form of the solution to the differential problem (Eqs. 18 and 20) is

$C(t, x)=\frac{1}{4 \pi t \sqrt{\operatorname{det} \boldsymbol{K}}} \exp \left[-\frac{\boldsymbol{x}^{T} \bullet \boldsymbol{K}^{-1} \bullet \boldsymbol{x}}{4 t}\right]$.

It is readily seen that the determinant of $\boldsymbol{K}$ is equal to unity, i.e.,

$\operatorname{det} \boldsymbol{K}=1$,

whereas its inverse is simply

$$
\boldsymbol{K}^{-1}=\left(\begin{array}{cc}
\cos ^{2} \theta+\alpha^{-2} \sin ^{2} \theta & -\left(\alpha^{-1}-\alpha\right) \sin \theta \cos \theta \\
-\left(\alpha^{-1}-\alpha\right) \sin \theta \cos \theta & \cos ^{2} \theta+\alpha^{2} \sin ^{2} \theta
\end{array}\right)
$$

Then, the solution may be rewritten as follows:

$C(t, x, z)=\frac{1}{4 \pi \mathrm{t}} \exp \left[\frac{-(\cos \theta x+\alpha \sin \theta z)^{2}-\left(\cos \theta z-\alpha^{-1} \sin \theta x\right)^{2}}{4 t}\right]$.

In the ocean, the slope of the isopycnal surfaces and the aspect ratio are usually small — which is why Cox (1987) suggested a simplified version of the isopycnal diffusivity tensor. For numerical experiments, the following values can be used (Mathieu and Deleersnijder 1998):

$\theta \approx 10^{-3} \approx \alpha$

The random walk model (Eq. 2) can now be cast into the following form:

$d X=\sqrt{2} V_{x x} d W_{1}$

$d Z=\sqrt{2} V_{x z} d W_{1}+\sqrt{2} V_{z z} d W_{2}$

$X(0)=Z(0)=0$,

where $\boldsymbol{K}=\boldsymbol{V} \boldsymbol{V}^{T}$ and the matrix $\boldsymbol{V}$ is

$\boldsymbol{V}=\left(\begin{array}{cc}V_{x x} & 0 \\ V_{x z} & V_{z z}\end{array}\right)=\left(\begin{array}{cc}\sqrt{K_{x x}} & 0 \\ \frac{K_{x z}}{\sqrt{K_{x x}}} & \sqrt{K_{z z}-\frac{K_{x z}^{2}}{K_{x x}}}\end{array}\right)$
To simulate the track of each particle, we use the Euler scheme (Eq. 48), i.e.,

$$
\begin{aligned}
& \bar{X}_{i+1}=\bar{X}_{i}+V_{x x} \sqrt{2 \Delta t} R_{i}^{(1)} \\
& \bar{Z}_{i+1}=\bar{Z}_{i}+\sqrt{2 \Delta t}\left(V_{x z} R_{i}^{(1)}+V_{z z} R_{i}^{(2)}\right) \\
& X_{0}=Z_{0}=0,
\end{aligned}
$$

where $i=0, \ldots, L-1, \Delta t=T / L$ and $R_{i}^{(1)}$ and $R_{i}^{(2)}$ are mutually independent variables with normal distribution with the parameters $N(0,1)$.

In our numerical simulation, we use the following parameters:

\begin{tabular}{lll} 
Aspect ratio & $\alpha$ & $10^{-3}$ \\
Slope of the isopycnal surface & $\theta$ & $10^{-3}$ \\
Time step & $\Delta t$ & $10^{-2}$ \\
Time of simulation & $T$ & 1 \\
Kernel function & $\mathcal{K}(\boldsymbol{u})$ & Epanechnikov \\
\hline
\end{tabular}

The exact solution is shown in Fig. 5, and the numerical solutions for the different numbers of particles are shown in Fig. 6. In Table 1, the results of the estimation of the concentration at the point are shown. For fixed number of 

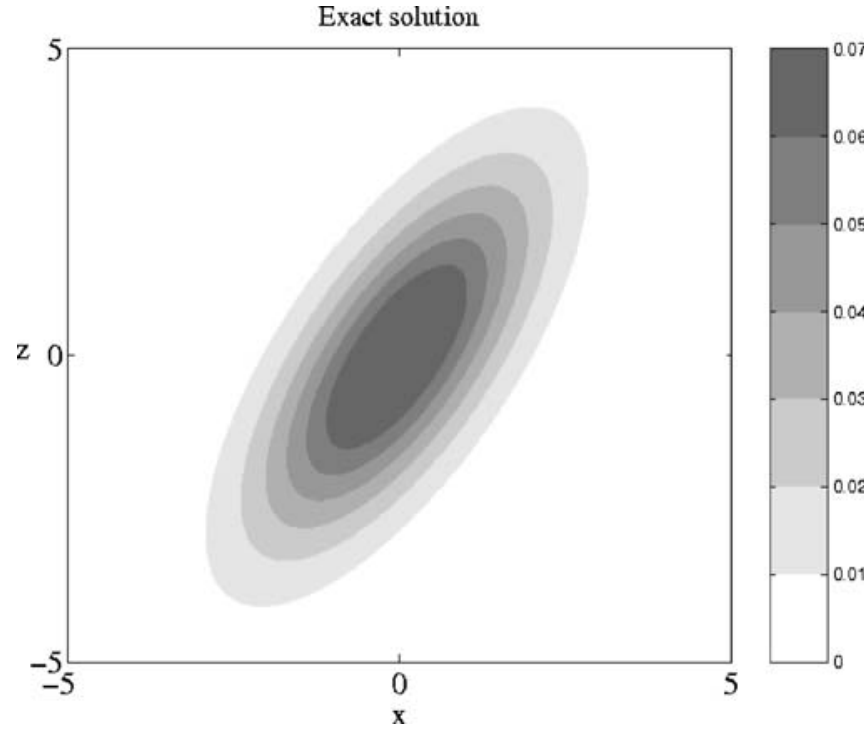

Fig. 5 The analytical solution of two-dimensional linear iso- and diapycnal advection-diffusion problem. The concentration is given by formula (Eq. 22)

particles $N$, we repeated the experiment several times to calculate the statistical error as follows:

$\varepsilon_{\text {statistical }}=\sqrt{\frac{1}{M-1} \sum_{m=1}^{M}\left(C^{(m)}(T, \boldsymbol{x})-\bar{C}(T, \boldsymbol{x})\right)^{2}}$,

where $M$ is the number of experiments (in our case the value $M=30$ was taken), $C^{(m)}, m=1, \ldots, M$ is the concentration obtained in the $m$ th simulation, and

$\bar{C}(T, \boldsymbol{x})=\frac{1}{M} \sum_{m=1}^{M} C^{(m)}(T, \boldsymbol{x})$

is the average value of concentration. The formula (Eq. 9) suggests that the rate of the convergence of $\varepsilon_{\text {statistical }}$ is supposed to be $\mathcal{O}\left(N^{-1 / 3}\right)$. In Fig. 7, it can be seen the statistical and the theoretical errors agree rather well with each other. It is clear that the statistical error has the same order of convergence as the theoretical error.

\section{Test problem 2: settling and diffusion model}

In the test case considered in the previous section, the area of interest is assumed to be infinite. However, in most applications, we have to deal with a bounded domain; that is the reason why it is important to test the random walk scheme for the model with space-varying diffusivity in the presence of boundaries. Therefore, we now consider a problem of settling and diffusion in a domain bounded by two boundaries (see Fig. 8). This model has been introduced and investigated in Deleersnijder et al. (2006a, b). Two special cases for which the exact solution is known are considered.

\subsection{Comparison with analytical solution}

Firstly, we consider the case when the settling velocity $w$ is zero. In this case, the concentration of the constituent under consideration can be derived from the following differential equation $(0 \leq t, 0 \leq z \leq h)$ :

$$
\begin{aligned}
& \frac{\partial C}{\partial t}=\frac{\partial}{\partial z}\left(k \frac{\partial C}{\partial z}\right) \\
& {\left[k \frac{\partial C}{\partial z}\right]_{z=0, h}=0} \\
& C(0, z)=\delta\left(z-z_{0}\right),
\end{aligned}
$$

where $k(z)$ is the eddy diffusivity. The latter is positive and nonzero in the interval $0<z<h$.

For the sake of generality, it is convenient to reformulate the problem above using dimensionless variables. The latter is defined to be

$t^{\prime}=\frac{t}{h^{2} / k}, \quad z^{\prime}=\frac{z}{h}, \quad k^{\prime}=\frac{k}{\bar{k}}$,

where $\bar{k}$ denotes the average over the domain of interest of the eddy diffusivity, i.e.,

$\bar{k}=h^{-1} \int_{0}^{h} k(z) d z$.

From here on, only dimensionless quantities will be used. This is why we will drop the primes. Therefore, using dimensionless variables, the domain of interest and the problem to be solved may be rewritten as follows:

$0 \leq t, \quad 0 \leq z \leq 1$,

$\frac{\partial C}{\partial t}=\frac{\partial}{\partial z}\left(k \frac{\partial C}{\partial z}\right)$

$\left[k \frac{\partial C}{\partial z}\right]_{z=0,1}=0$

$C(0, z)=\delta\left(z-z_{0}\right)$,

where the domain-averaged values of the eddy diffusivity must be equal to unity, i.e.,

$\bar{k}=\int_{0}^{h} k(z) d z=1$.

For the purposes of the present study, it is convenient to consider that the eddy diffusivity does not depend on time, but further assuming that it is also independent of the vertical coordinate would be too strong an idealization (Burchard 

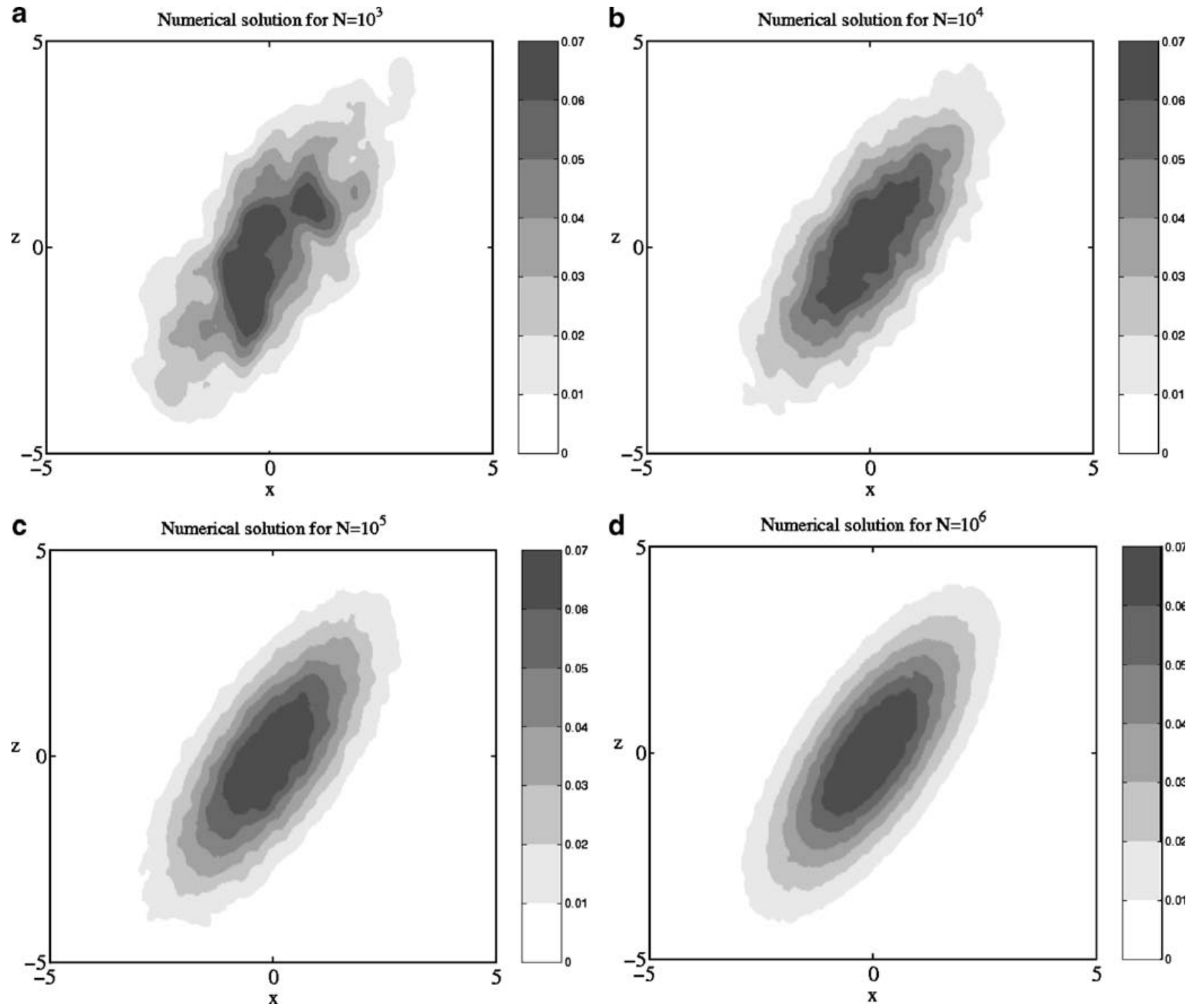

Fig. 6 The numerical solution of two-dimensional linear iso- and diapycnal advection-diffusion problem obtained by applying the random walk model (Eq. 24) for a $10^{3}$ particles, b $10^{4}$ particles, c $10^{5}$ particles, and $\mathbf{d} 10^{6}$ particles

2002; Umlauf and Burchard 2005). As an example of the diffusivity profile, the following function may be chosen

$k(z)=6 z(1-z)$.

Table 1 The concentration at the location $(1.8,2.5)$

\begin{tabular}{llll}
\hline$N$ & Average value & Statistical error & Exact \\
\hline $10^{3}$ & 0.0295 & 0.0067 & 0.0313 \\
$5 * 10^{3}$ & 0.0313 & 0.0038 & 0.0313 \\
$10^{4}$ & 0.0308 & 0.0028 & 0.0313 \\
$5 * 10^{4}$ & 0.0303 & 0.0020 & 0.0313 \\
$10^{5}$ & 0.0312 & 0.0013 & 0.0313 \\
$5 * 10^{5}$ & 0.0314 & 0.0007 & 0.0313 \\
$10^{6}$ & 0.0312 & 0.0006 & 0.0313 \\
\hline
\end{tabular}

This choice is consistent with the diffusion processes in the upper mixed layer. The diffusivity profile $k(z)$ tends to zero as the bottom of the mixed layer is approached, and the maximum of the diffusivity should not occur at the surface (Deleersnijder et al. 2006a). It is clear that the diffusivity tensor (Eq. 32) satisfies these conditions. It can be shown that, in this case, the analytical solution can be obtained in the form of

$$
\begin{aligned}
& C(t, z)=1+ \\
& \sum_{n=1}^{\infty}(2 n+1) P_{n}(2 z-1) P_{n}\left(2 z_{0}-1\right) e^{-6 n(n+1) t},
\end{aligned}
$$

where $P_{n}(z)$ denotes the $n$-th order Legendre polynomial. Figure 9 presents the analytical solution for the different moments of time obtained for $z_{0}=1 / 2$. 


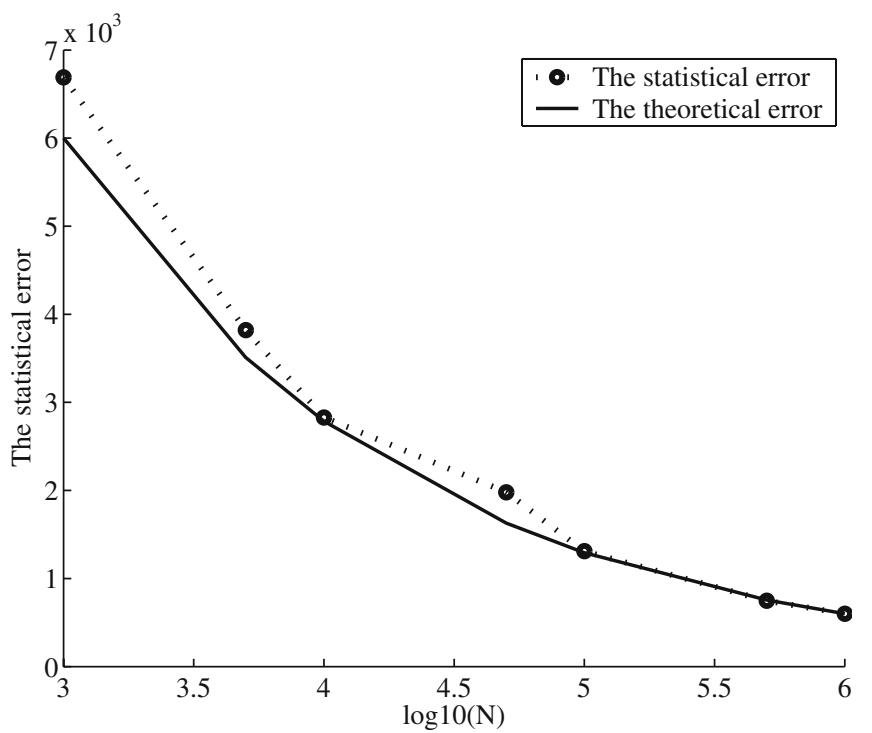

Fig. 7 The comparison of the statistical error with the theoretical error in the case of the two-dimensional advection-diffusion problem (see Table 1)

The random walk model (Eq. 2) in this case has the following form:

$$
\begin{aligned}
& d Z(t)=\frac{\partial k}{\partial z} d t+\sqrt{2 k(z)} d W(t) \\
& Z(0)=z_{0} .
\end{aligned}
$$

For the numerical solution (see Figs. 10 and 11), we apply the Euler scheme (Eq. 48), which, in this case, can be written in the following form (with time step $\Delta t=3 \cdot 10^{-5}$ )

$$
\begin{aligned}
& \bar{Z}_{i+1}=6\left(1-2 \bar{Z}_{i}\right) \Delta t+\sqrt{12 \Delta t \bar{Z}_{i}\left(1-\bar{Z}_{i}\right)} R_{i} \\
& \bar{Z}_{0}=z_{0}
\end{aligned}
$$

where $i=0, \ldots, L-1, \Delta t=t / L$, and $R_{i}$ are mutually independent random variables with normal distribution with parameters $N(0,1)$. For this example, the kernel estimator (Eq. 3) with the Gaussian kernel (Eq. 4) was

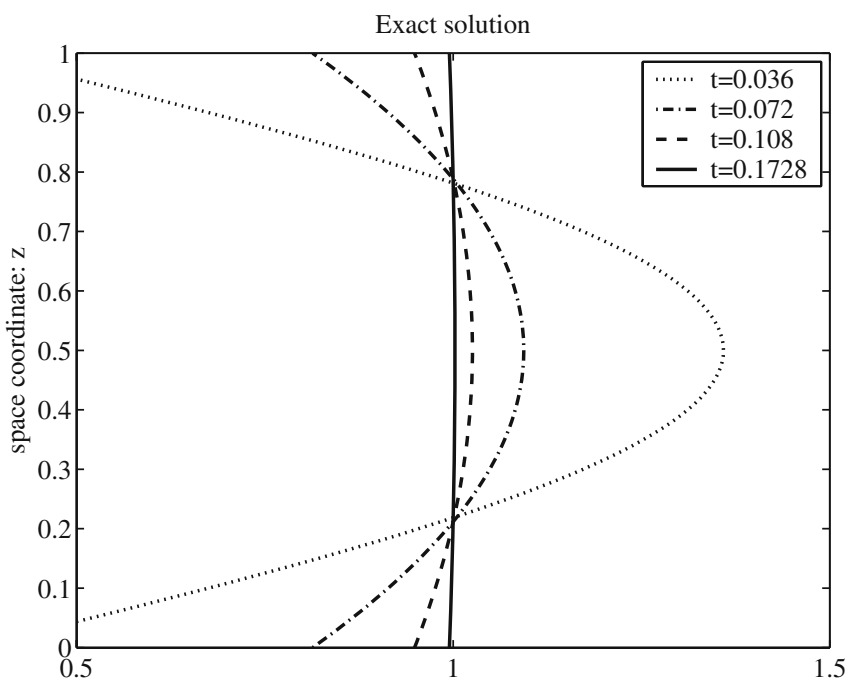

Fig. 9 The concentration profile for different moments of the simulation, obtained by using the explicit representation (Eq. 33). All variables are dimensionless

compared with the box-counting method. Figure 10 illustrates the results of the kernel estimator with the following choice of bandwidth:

$$
\begin{aligned}
& \lambda=K N^{-\frac{1}{5}}, \\
& K=\sqrt{\frac{1}{N-1} \sum_{j=1}^{N}\left(\bar{Z}^{(j)}(t)-\frac{1}{N} \sum_{l=1}^{N} \bar{Z}^{(l)}(t)\right)^{2}},
\end{aligned}
$$

where $K$ is the standard deviation of the sample $\bar{Z}^{(i)}, i=1, \ldots, N$.

The numerical solution shown in Fig. 11 was obtained as a result of the box-counting approach. The domain was divided into 50 intervals, so the size of each interval is 0.02 . It is clear that the solution obtained by using the kernel estimator is more accurate than by using the box-counting approach at least by one order of magnitude.

We calculated the concentration at the middle of the boundary layer and at the boundary (using the kernel
Fig. 8 Sinking-diffusion model: illustration of its geometry, parameters, and boundary conditions. Source: Deleersnijder et al. (2006a)

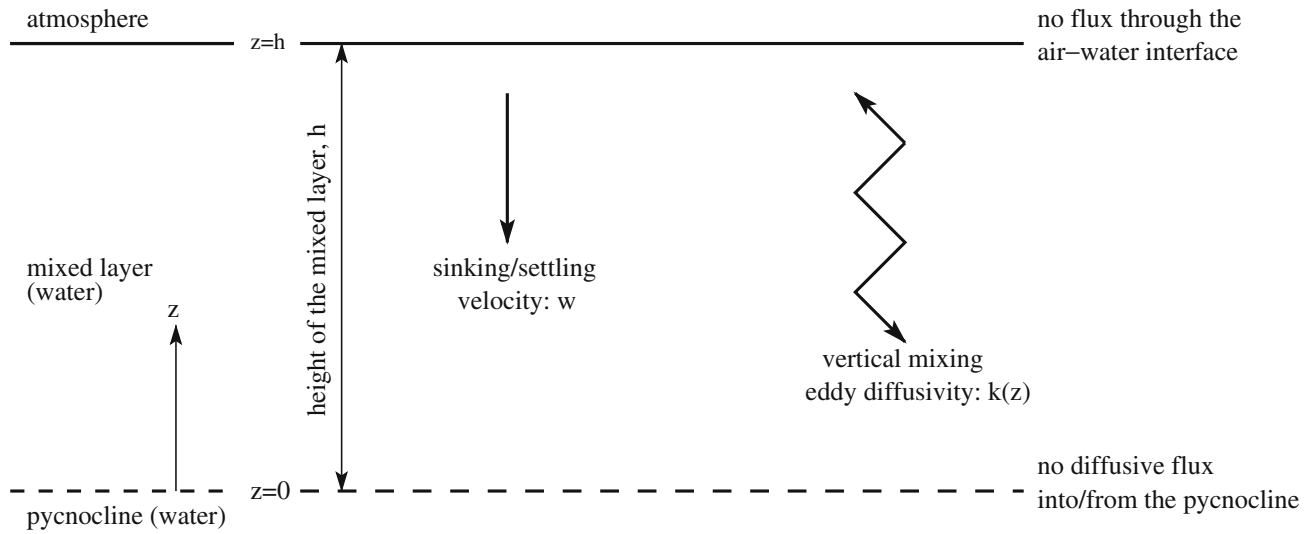



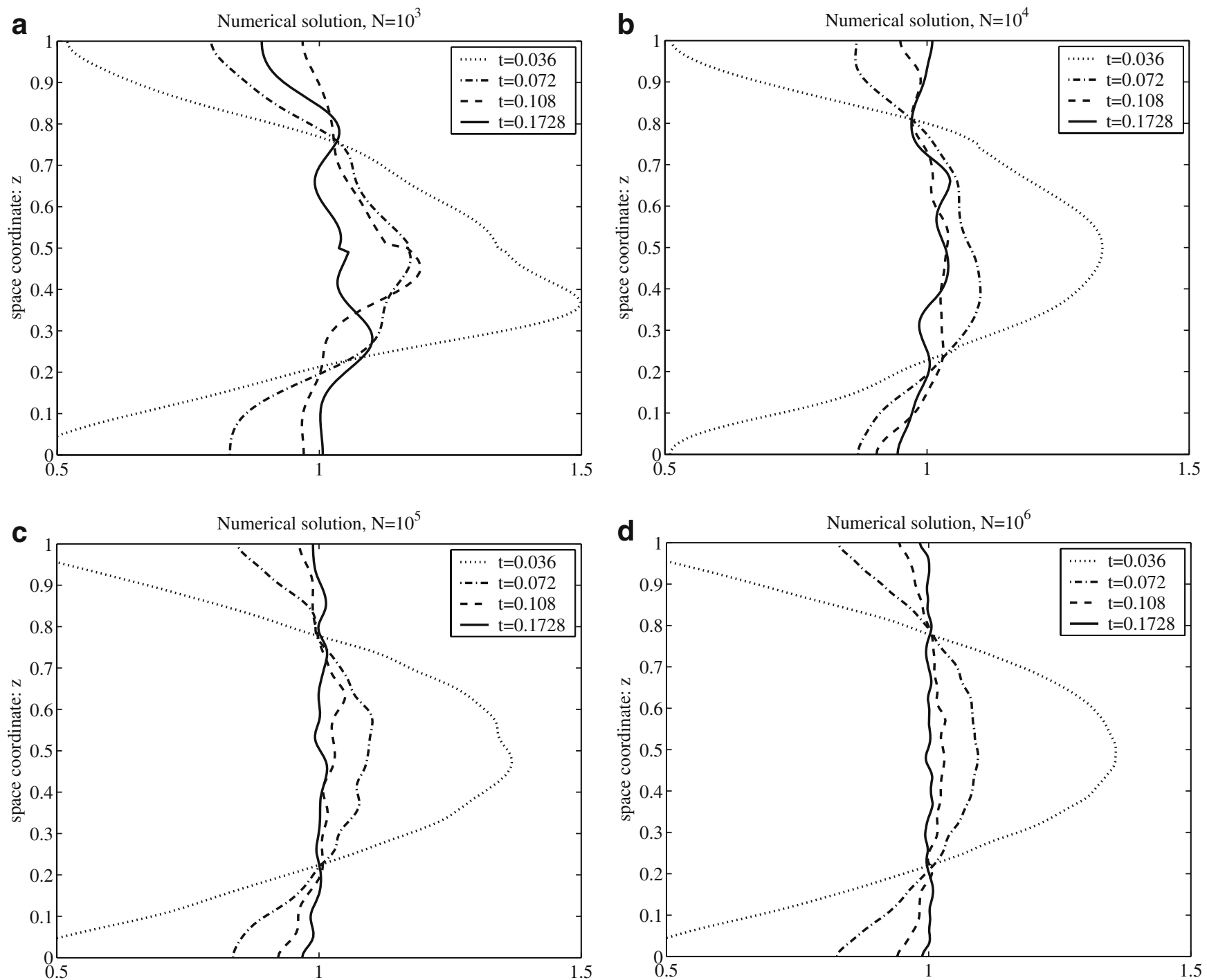

Fig. 10 The numerical approximation of the concentration profile for the eddy diffusivity function (Eq. 32) for a $N=10^{3}, \mathbf{b} N=10^{4}$, c $N=10^{5}$, and $\mathbf{d} N=10^{6}$. The kernel estimator with the Gaussian kernel (Eq. 4) was used

estimator) and repeated each simulation 30 times to find the statistical error. The results for different moments of time and different number of particles are presented in Table 2. In Tables 3 and 4, one can see the concentration calculated at the boundary $z=0$ using the reflection method (Table 3) and the boundary kernel (Eq. 8; Table 4).

In Section 2, it was mentioned that the reflection of the kernel function near the boundary allows to achieve the consistency with the true value of the concentration but still has a large bias of order $\mathcal{O}(\lambda)$. It can be seen from Eq. 7 that the bias is proportional to the concentration gradient. When the concentration gradient at the boundary is high (for instance, for $t=0.018$ or $t=0.036$ ), one can see from Table 3 that the estimated concentration is larger than the true value of the concentration. For larger $N$, the optimal bandwidth $\lambda=\mathcal{O}\left(N^{-1 / 5}\right)$ becomes smaller, and the numerical concentration converges to the true value.

An alternative to the reflection of the kernel is the boundary kernel. In this case, the average value of the concentration calculated by Eq. 8 is not affected by systematic errors. However, we cannot guarantee the positiveness of the solution anymore. Furthermore, the uncertainty of the numerical solution is higher near the boundary than at the interior point (see Table 4).

\subsection{The residence time}

If the settling velocity $w$ is nonzero, the analytical solution cannot be obtained. However, the exact solution for the adjoint problem of finding the residence time $\theta\left(z_{0}\right)$ is known (Bolin and Rodhe 1973; Delhez et al. 2004; Deleersnijder et al. 2006a, b). To obtain the residence time, $\theta\left(z_{0}\right)$, a unit amount of tracer, is released at the initial time at a distance $z_{0}$ to the pycnocline. In other words, the initial condition reads

$C(0, z)=\delta\left(z-z_{0}\right)$. 

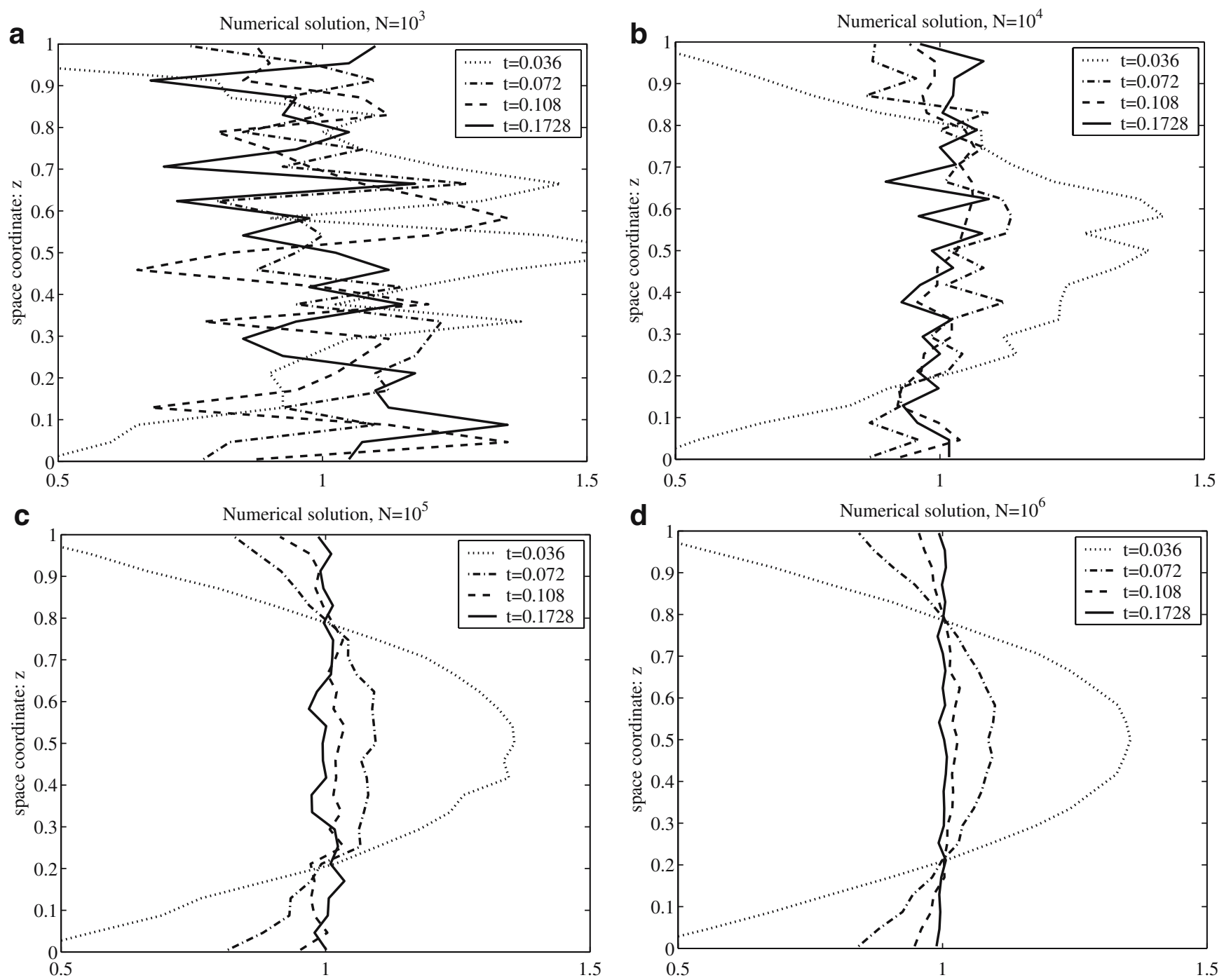

Fig. 11 The numerical approximation of the concentration profile for the eddy diffusivity function (Eq. 32) for a $N=10^{3}, \mathbf{b} N=10^{4}$, c $N=10^{5}$, and $\mathbf{d} N=10^{6}$. The box estimator was used

As it has been done in the previous examples, it is convenient to introduce dimensionless variables:

$t^{\prime}=\frac{t}{h / w}, \quad\left(z^{\prime}, z_{0}^{\prime}\right)=\frac{\left(z, z_{0}\right)}{h}, \quad k^{\prime}=\frac{k}{\bar{k}}, \quad \theta^{\prime}=\frac{\theta}{h / w} . \quad$ (38) $\quad P e=\frac{w h}{\bar{k}}$

Table 2 The exact and numerical concentration and the statistical error at the location $z=0.5$ (middle of the boundary layer)

\begin{tabular}{lllll}
\hline Time $t$ & $N=10^{3}$ & $N=10^{4}$ & $N=10^{5}$ & Exact \\
\hline 0.0036 & $3.762 \pm 0.192$ & $3.856 \pm 0.053$ & $3.873 \pm 0.011$ & 3.881 \\
0.018 & $1.812 \pm 0.087$ & $1.799 \pm 0.031$ & $1.811 \pm 0.013$ & 1.814 \\
0.036 & $1.326 \pm 0.074$ & $1.356 \pm 0.026$ & $1.359 \pm 0.010$ & 1.359 \\
0.054 & $1.163 \pm 0.055$ & $1.174 \pm 0.029$ & $1.180 \pm 0.012$ & 1.181 \\
0.072 & $1.079 \pm 0.058$ & $1.089 \pm 0.024$ & $1.092 \pm 0.009$ & 1.094 \\
0.108 & $1.025 \pm 0.060$ & $1.020 \pm 0.021$ & $1.000 \pm 0.008$ & 1.023 \\
0.172 & $0.990 \pm 0.063$ & $1.003 \pm 0.025$ & 1.003 \\
\hline
\end{tabular}

All variables are dimensionless. 
Table 3 The exact and numerical concentration and the statistical error at the location $z=0.0$ (the boundary)

\begin{tabular}{lllll}
\hline Time $t$ & $N=10^{3}$ & $N=10^{4}$ & $N=10^{5}$ & Exact \\
\hline 0.018 & $0.134 \pm 0.034$ & $0.097 \pm 0.014$ & $0.074 \pm 0.004$ & 0.040 \\
0.036 & $0.502 \pm 0.057$ & $0.465 \pm 0.017$ & $0.425 \pm 0.009$ & 0.360 \\
0.054 & $0.735 \pm 0.068$ & $0.712 \pm 0.032$ & $0.689 \pm 0.011$ & 0.647 \\
0.072 & $0.883 \pm 0.053$ & $0.844 \pm 0.030$ & $0.839 \pm 0.014$ & 0.813 \\
0.108 & $0.970 \pm 0.078$ & $0.953 \pm 0.029$ & $0.950 \pm 0.013$ & 0.949 \\
0.172 & $1.003 \pm 0.098$ & $0.995 \pm 0.036$ & $0.992 \pm 0.013$ & 0.995 \\
\hline
\end{tabular}

All variables are dimensionless. The kernel estimator was transformed near the boundary by reflection using the reflection technique based on the Epanechnikov kernel (see Section 2).

and because only the dimensionless variables will be used from now on, we will drop all primes. Accordingly, the domain of the interest is now defined as $0 \leq t$ and $0 \leq z \leq 1$.

In Deleersnijder et al. (2006a), it is shown that the residence time corresponding to the parabolic profile (Eq. 32; Fig. 12a) is

$\theta(z)=z+\left(\frac{z}{1-z}\right)^{\mu} B_{1-z}(1+\mu, 1-\mu)$

where $B_{1-z}(1+\mu, 1-\mu)$ is a generalized incomplete beta function, i.e.,

$B_{1-z}(1+\mu, 1-\mu)=\int_{0}^{1-z} \sigma^{\mu}(1-\sigma)^{-\mu} d \sigma$

and $\mu=P e / 6$. by

In this test problem, the random walk algorithm is given

$d Z(t)=\left(w+\frac{\partial k}{\partial z}\right) d t+\sqrt{2 k(z)} d W(t)$

$Z(0)=z_{0}$.

We release particles at the position $z_{0}$ and model their movement using a Euler scheme similar to Eq. 35. For each realization of the particle track, we calculate the residence time, that is, how much time the particle needs to leave the domain. By averaging the results, the average residence time can be obtained. The numerical results for $N=10^{3}$, $N=10^{4}$ and $N=10^{5}$ are shown in Fig. 12b-d. For this numerical simulation, the following parameters were used:

\begin{tabular}{lll}
\hline Time step & $\Delta t$ & $10^{-4}$ \\
Peclet number & $P e$ & 5. \\
\hline
\end{tabular}

Comparing the exact solution (Fig. 12a) with the numerical solutions (Fig. 12b-d), it is clear that the numerical solution for $N=10^{3}$ particles already provides a good approximation of the exact solution.

\section{Conclusion}

In this paper, the random walk model for the simulation of diffusion processes with space-varying diffusivity is introduced and analyzed. The kernel estimator is applied instead of the traditional box-counting method. It is shown that the kernel estimator allows to reduce the number of particles by one order of magnitude as compared with the box-counting method. This Lagrangian model is applied to several test problems, and results show that this random walk model may be a good alternative to commonly used Eulerian models, for instance, in the case of a spacevarying, nondiagonal diffusivity matrix.

Table 4 The exact and numerical concentration and the statistical error at the location $z=0.0$ (the boundary)

\begin{tabular}{lllll}
\hline Time $t$ & $N=10^{3}$ & $N=10^{4}$ & $N=10^{5}$ & Exact \\
\hline 0.018 & $0.072 \pm 0.095$ & $0.031 \pm 0.028$ & $0.040 \pm 0.012$ & 0.040 \\
0.036 & $0.348 \pm 0.204$ & $0.372 \pm 0.065$ & $0.366 \pm 0.027$ & 0.360 \\
0.054 & $0.695 \pm 0.192$ & $0.641 \pm 0.102$ & $0.640 \pm 0.027$ & 0.647 \\
0.072 & $0.844 \pm 0.247$ & $0.826 \pm 0.136$ & $0.802 \pm 0.042$ & 0.813 \\
0.108 & $0.980 \pm 0.270$ & $0.993 \pm 0.096$ & $0.954 \pm 0.055$ & 0.949 \\
0.172 & $1.065 \pm 0.307$ & $0.983 \pm 0.100$ & $0.986 \pm 0.049$ & 0.995 \\
\hline
\end{tabular}

All variables are dimensionless. The kernel boundary (Eq. 8) was used. 

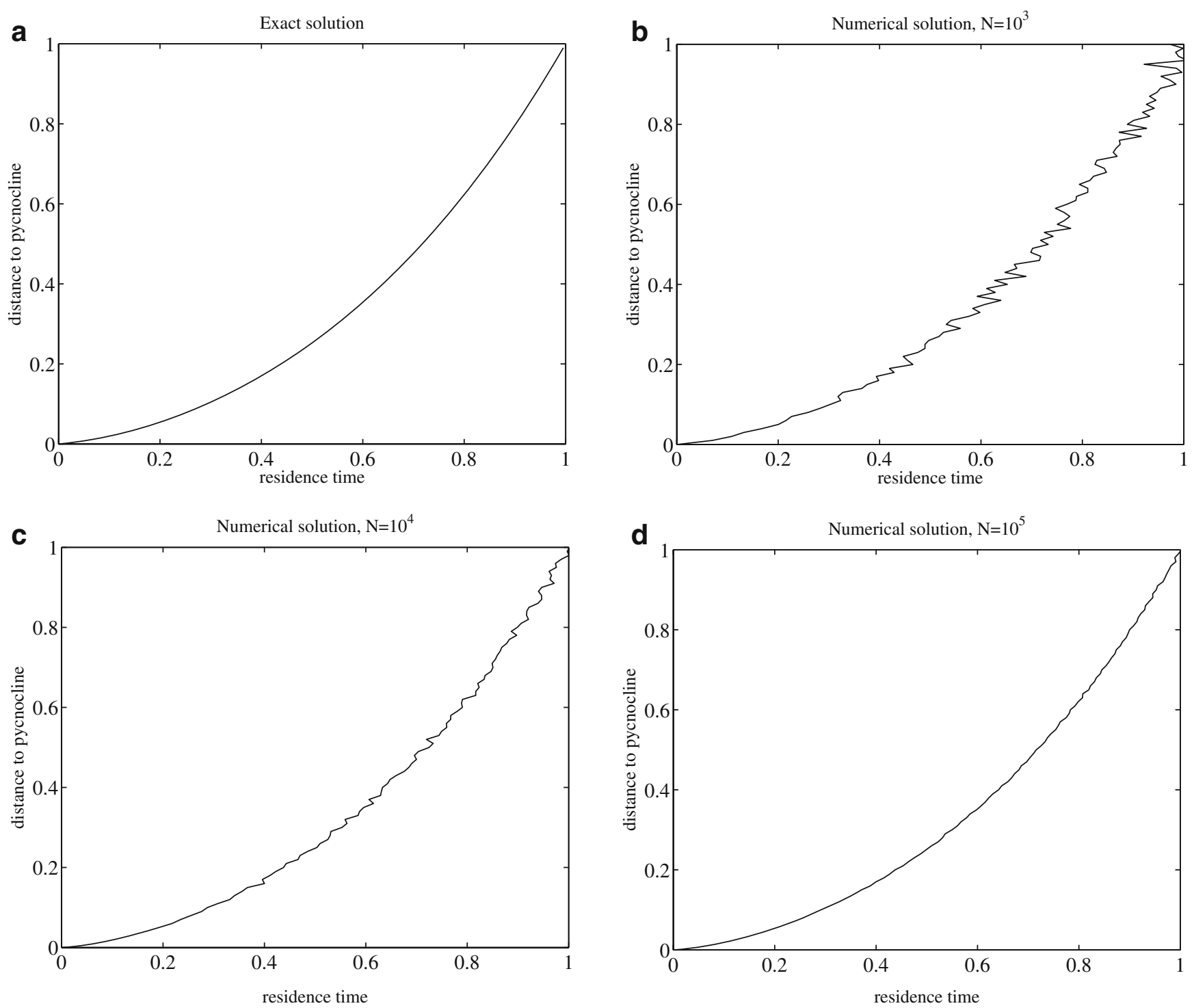

Fig. 12 The residence time for the diffusivity profile (Eq. 31): exact (a) and numerical approximation for $\mathbf{b} N=10^{3}$, c $N=10^{4}$, and d $N=10^{5}$ particles

\section{A Appendix: SDE}

A lot of natural phenomena in physics, control theory, biology, economics, and other areas are influenced by some random disturbances. Therefore, for their modelling, we need to use a differential equation with the stochastic term

$\frac{d X(t)}{d t}=a(t, X(t))+\sigma(t, X(t)) \cdot{ }^{\prime \prime}$ noise"

The theory of SDEs has been developed to handle equations like Eq. 43 with a 'noise' term. One of the methods to define this equation in a proper way is to use the Brownian motion (Wiener process). The Brownian motion $W(t), t \in$ $[0, T]$ is called the Gaussian stochastic process (it means that for any $0 \leq t_{1} \leq \ldots \leq t_{k} \leq T$, the random variable $\boldsymbol{Z}=\left(W\left(t_{1}\right), W\left(t_{2}\right), \ldots, W\left(t_{k}\right)\right) \in \mathbb{R}^{k}$ has a normal distribution (Oksendal 1985) with the following statistics:

$$
\begin{aligned}
& E\left(W\left(t_{2}\right)-W\left(t_{1}\right)\right)=0 \\
& E\left(\left(W\left(t_{4}\right)-W\left(t_{3}\right)\right)\left(W\left(t_{2}\right)-W\left(t_{1}\right)\right)\right)=0, \\
& \quad t_{4} \geq t_{3} \geq t_{2} \geq t_{1} \\
& E\left(\left(W\left(t_{2}\right)-W\left(t_{1}\right)\right)\left(W\left(t_{2}\right)-W\left(t_{1}\right)\right)\right)=\left(t_{2}-t_{1}\right), \\
& \quad t_{2} \geq t_{1} .
\end{aligned}
$$


The stochastic process $W(t)$ represents the "noise" term, and the equation (Eq. 43) can be rewritten in the form (with the initial condition $X(0)=x_{0}$ )

$$
\begin{aligned}
& d X(t)=a(t, X(t)) d t+\sigma(t, X(t)) d W(t) \\
& X(0)=x_{0} .
\end{aligned}
$$

The equation (Eq. 45) is called SDE in Îto sense. This equation can be easily extended to a system of the SDEs (Arnold 1974; Oksendal 1985; Jazwinski 1970):

$$
\begin{aligned}
& d \boldsymbol{X}(t)=\boldsymbol{a}(t, \boldsymbol{X}(t)) d t+\boldsymbol{\sigma}(t, \boldsymbol{X}(t)) d \boldsymbol{W}(t) \\
& \boldsymbol{X}(0)=\boldsymbol{x}_{0},
\end{aligned}
$$

where $\boldsymbol{X} \in \mathbb{I}^{d}$ is a $d$-dimensional stochastic process, $\boldsymbol{a}$ is a $d$-dimensional vector function and $\sigma$ is $d \times m$-matrix function, and $m$-dimensional Brownian motion process $\boldsymbol{W}$ has the following statistics

$$
\begin{aligned}
& E\left(\boldsymbol{W}\left(t_{2}\right)-\boldsymbol{W}\left(t_{1}\right)\right)=0 \\
& E\left(\left(\boldsymbol{W}\left(t_{4}\right)-\boldsymbol{W}\left(t_{3}\right)\right)\left(\boldsymbol{W}\left(t_{2}\right)-\boldsymbol{W}\left(t_{1}\right)\right)\right)=0, \\
& t_{4} \geq t_{3} \geq t_{2} \geq t_{1} \\
& E\left(\left(\boldsymbol{W}\left(t_{2}\right)-\boldsymbol{W}\left(t_{1}\right)\right)\left(\boldsymbol{W}\left(t_{2}\right)-\boldsymbol{W}\left(t_{1}\right)\right)\right)=\left(t_{2}-t_{1}\right) \boldsymbol{I}_{m}, \\
& t_{2} \geq t_{1},
\end{aligned}
$$

where $\boldsymbol{I}_{m}$ is $m \times m$ identity matrix.

Furthermore, we will assume that the functions $\boldsymbol{a}$ and $\sigma$ are continuously differentiable, and the matrix $\boldsymbol{b}:=\boldsymbol{\sigma} \boldsymbol{\sigma}^{T}$ is of full rank for every $(t, \boldsymbol{x}) \in[t, T] \times \mathbb{I R}^{d}$. This particularly implies the existence and uniqueness of the solution of Eq. 46. Sometimes it is impossible to solve the SDE directly, but we can find the probability laws of the random variable $\boldsymbol{X}(t)$ for any fixed moment of time $t$. In this case, we speak about the solution in the weak sense (Arnold 1974; Jazwinski 1970; Oksendal 1985). Roughly speaking, for the strong solution, it is important to find the realization of the function $\boldsymbol{X}(t)$, and for the weak solution, it is important to determine only the probability law of the random variable $\boldsymbol{X}(t)$. Hereinafter, we will consider only weak solutions.

In a general case, it is impossible to find the analytical solution of the SDE (Eq. 46), and we have to use one of the numerical schemes. The simplest and most often used method is the Euler scheme (Kloeden and Platen 1992; Milstein and Tretyakov 2004) that is a one-step approximation method of following form:

$\overline{\boldsymbol{X}}_{i+1}^{\Delta t}=\overline{\boldsymbol{X}}_{i}^{\Delta t}+\boldsymbol{a}\left(t, \overline{\boldsymbol{X}}_{i}^{\Delta t}\right) \Delta t+\boldsymbol{\sigma}\left(t, \overline{\boldsymbol{X}}_{i}^{\Delta t}\right) \Delta \boldsymbol{W}_{i}$,

$\bar{X}_{0}^{\Delta t}=\boldsymbol{x}_{0}$,

where $i=0, \ldots, L-1, \overline{\boldsymbol{X}}_{i}^{\Delta t}:=\overline{\boldsymbol{X}}^{\Delta t}\left(t_{i}\right)$ is the numerical approximation of the position $\boldsymbol{X}\left(t_{i}\right), t_{i}=i \Delta t, \Delta t=t / L$ is the time step of numerical integration, and $\Delta \boldsymbol{W}_{i}$ are mutually independent Gaussian variables with zero mean and covariance matrix $\Delta t \boldsymbol{I}_{m}$.

To determine if one or the other method is suitable for us, we need to define the criteria of accuracy of the method. We shall say that an approximation $\overline{\boldsymbol{X}}^{\Delta t}$ converges to the solution $\boldsymbol{X}$ of Eq. 46 at time $T$ with order $\beta>0$ (strictly speaking, we should say with weak order) if for each positive polynomial $g$ exists a positive constant $K$, which does not depend on $\Delta t$ such that

$$
\left|E(g(\boldsymbol{X}(T)))-E\left(g\left(\overline{\boldsymbol{X}}^{\Delta t}(T)\right)\right)\right| \leq K(\Delta t)^{\beta} .
$$

The Euler scheme converges with weak order $\beta=1$, which sometimes is not enough for the accurate numerical solution of Eq. 46. We can obtain a higher order accuracy from a one-step scheme by an extrapolation method. We introduce now the extrapolation method for the simulation of the functional stochastic process $\boldsymbol{X}$ based on the Euler approximation assuming that the function $g$ and its derivatives are smooth. First, one should use the Euler approximation $\overline{\boldsymbol{X}}^{\Delta t}$ generated by Eq. 48 with step size $\Delta t$ and simulate the functional

$U^{\Delta t}=E\left(g\left(\overline{\boldsymbol{X}}^{\Delta t}(T)\right)\right)$,

then find the functional for the simulation with double time step

$U^{2 \Delta t}=E\left(g\left(\overline{\boldsymbol{X}}^{2 \Delta t}(T)\right)\right)$.

Finally, two results are combined to yield the approximation

$\bar{U}^{\Delta t}=2 U^{\Delta t}-U^{2 \Delta t}$.

In this way, we obtain from the first weak order $\beta=1.0$ Euler scheme a method of weak order $\beta=2.0$. This method is the extension of the Romberg or Richardson method for partial differential equations, which was proposed by Talay and Tubaro (1991).

The SDEs can be used for the solution of ordinary differential equations. For instance, the connection between the boundary value problem of parabolic type and the corresponding SDE is given by the Feynman-Kac formula (Oksendal 1985). However, for the solution of the diffusion equation, we need only to consider the so-called Fokker-Planck equation.

Suppose that the transition density function $p(t, \boldsymbol{x} ; s, \boldsymbol{y})$ is continuous with respect to $t$ and $s$, and all first and second derivatives with respect to $\boldsymbol{x}$ and $\boldsymbol{y}$ exist and are 
continuous, then $p(t, \boldsymbol{x} ; s, \boldsymbol{y})$ is the solution of the FokkerPlanck equation $(0 \leq t \leq s \leq T)$

$$
\begin{aligned}
& \frac{\partial p}{\partial s}+\sum_{i=1}^{d} \frac{\partial}{y_{i}}\left(a_{i}(s, \boldsymbol{y}) p\right)-\frac{1}{2} \sum_{i, j=1}^{d} \frac{\partial^{2}}{\partial y_{i} \partial y_{j}}\left(b_{i j}(s, \boldsymbol{y}) p\right)=0, \\
& p(t, \boldsymbol{x} ; t, \boldsymbol{y})=\delta(\boldsymbol{x}-\boldsymbol{y}) .
\end{aligned}
$$

\section{References}

Arnold L (1974) Stochastic differential equations. Wiley, New York Beckers JM, Burchard H, Campin JM, Deleersnijder E, Mathieu PP (1998) Another reason why simple discretizations of rotated diffusion operators cause problems in ocean models: comments on "Isoneutral diffusion in a $z$-coordinate ocean model". J Phys Oceanogr 28:1552-1559

Beckers JM, Burchard H, Deleersnijder E, Mathieu PP (2000) Numerical discretization of rotated diffusion operators in ocean models. Mon Weather Rev 128:2711-2733

Bolin B, Rodhe H (1973) A note on concepts of age distribution and transit time in natural reservoirs. Tellus 25:58-62

Burchard H (2002) Applied turbulence modelling in marine waters. Lecture notes in earth sciences, vol 100. Springer, Berlin Heidelberg New York

Celia MA, Russell TF, Herrera I, Ewing RE (1990) An EulerianLagrangian localized adjoint method for an advection-diffusion equation. Adv Water Resour 13(4):187-206

Cox MD (1987) Isopycnal diffusion in a $z$-coordinate model. Ocean Model 74:1-5

Deleersnijder E, Camin JM, Delhez EJM (2001) The concept of age in marine modelling. I. Theory and preliminary model results. J Mar Syst 28:229-267

Deleersnijder E, Beckers JM, Delhez EJM (2006a) The residence time of settling in the surface mixed layer. Environ Fluid Mech 6(1):25-42

Deleersnijder E, Beckers JM, Delhez EJM (2006b) On the behaviour of the residence time at bottom of the mixed layer. Environ Fluid Mech 6:541-547

Delhez EJM, Heemink AW, Deleersnijder E (2004) Residence time in a semi-enclosed domain from the solution of an adjoint problem. Estuar Coast Shelf Sci 61:691-702

de Haan P (1999) On the use of density kernels for concentration estimations within particles and puff dispersion models. Atmos Environ 33:2007-2021

Dimou KN, Adams EE (1993) A random-walk, particles tracking models for well-mixed estuaries and coastal waters. Estuar Coast Shelf Sci 37:99-110

Jazwinski AW (1970) Stochastic differential processes and filtering theory. Academic, New York

Jones MC (1993) Simple boundary correction for kernel density estimation. Stat Comput 3:135-146

Heemink AW (1990) Stochastic modeling of dispersion in shallow water. Stoch Hydrol Hydraul 4:161-174

Kinzelbach W (1988) The random walk method in pollutant transport simulation. In: Custodio et al (eds) Groundwater flow and quality modeling. Reidel, Dordrecht, pp 227-245

Kloeden PE, Platen E (1992) Numerical solution of stochastic differential equations. Springer, Berlin Heidelberg New York

Konikow LF, Bredehoeft JD (1978) Computer model of twodimensional solute transport and dispersion in ground water. Techniques of water-resources investigations of the US Geological Survey, chapter C2, book 7, US Government Printing Office, Washington DC

LaBolle EM, Fogg GE, Tompson AFB (1996) Random-walk simulation of transport in heterogeneous porous media: local mass-conservation problem and implementation methods. Water Resour Res 32(3):583-593
Mathieu PP, Deleersnijder E (1998) What is wrong with isopycnal diffusion in world ocean models? Appl Math Model 22:367-378

Mathieu PP, Deleersnijder E, Beckers JM (1999) Accuracy and stability of the discretised isopycnal-mixing equation. Appl Math Lett $12: 81-88$

Milstein GN, Tretyakov MV (2004) Stochastic numerics for mathematical physics. Springer, Berlin Heidelberg New York

Müller HG (1991) Smooth optimum kernel estimators near endpoints. Biometrika 78(3):521-530

Proehl JA, Lynch DE, McGillicuddy Jr DJ, Ledwell JR (2005) Modelling turbulent dispersion of the North Flank of Georges Bank using Lagrangian methods. Cont Shelf Res 25:875-900

Oksendal B (1985) Stochastic differential equations. Springer, Berlin Heidelberg New York

Redi MH (1982) Oceanic isopycnal mixing by coordinate rotation. J Phys Oceanogr 12:1154-1158

Riddle AM (1998) The specification of mixing in random walk models for dispersion in the sea. Cont Shelf Res 18:441-456

Sawford B (2001) Turbulent relative dispersion. Annu Rev Fluid Mech 33:289-317

Schoenmakers JGM, Heemink AW (1997) Fast valuation of financial derivatives. J Comput Financ 1:47-62

Silverman BW (1986) Density estimation for statistics and data analysis. Chapman and Hall, London

Spivakovskaya D, Heemink AW, Milstein GN, Schoenmakers JGM (2005) Simulation of the transport of particles in coastal waters using forward and reverse time diffusion. Adv Water Resour 28:927-938

Stijnen JW (2002) Numerical methods for stochastic environmental models. Ph.D. dissertation, Delft University of Technology, The Netherlands

Sun NZ (1999) A finite cell method for simulating the mass transport process in porous media. Water Resour Res 35(12):3649-3662

Talay D, Tubaro L (1991) Expansion of the global error for numerical schemes solving stochastic differential equations. Stoch Anal Appl 8(4):485-509

Thomson DJ (1987) Criteria for the selection of stochastic models of particles trajectories in turbulent flow. J Fluid Mech 180:529-556

Uffink GJM (1988) Modeling of solute transport with the random walk method. In: Custodio et al (eds) Groundwater flow and quality modeling, Reidel, Dordrecht, pp 247-265

Umlauf L, Burchard H (2005) Second order turbulence closure models for geophysical boundary layers. A review of recent work. Cont Shelf Res 25:795-827

van Stijn ThL, Praagman N, van Eijkeren J (1987) Positive advection schemes for environmental studies. In: Taylor $\mathrm{C}$ et al. (eds) Numerical methods in laminar and turbulent flow. Pineridge, Swansea, pp 1256-1267

Wand MP, Jones MC (1995) Kernel smoothing. Chapman and Hall, London

Yang Y, Wilson LT, Makela ME, Marchetti MA (1998) Accuracy of numerical methods for solving the advection-diffusion equation as applied to spore and insect dispersal. Ecol Model 109:1-24

Yeh GT (1990) A Lagrangian-Eulerian method with zoomable hidden fine-mesh approach to solving advection-dispersion equations. Water Resour Res 26(6):1133-1144

Zhang R, Huang K, van Geruchten MT (1993) An efficient EulerianLagrangian method for solving solute transport problems in steady and transient flow fields. Water Resour Res 28(12):4131-4138

Zheng C, Bennett GD (2002) Applied contaminant transport modeling. Wiley, New York

Zheng C, Wang PP (1999) MT3DMS: a modular three-dimensional multispecies transport model for simulation of advection, dispersion and chemical reactions of contaminants in groundwater systems; documentation and user's guide, contract report SERDP-99-1. US Army engineer research and development center, Vicksburg, MS

Zimmermann S, Koumoutsakos P, Kinzelbach W (2001) Simulation of pollutant transport using a particle method. J Comput Phys 173(1):322-347 